Pacific

Journal of

Mathematics

THE IRREDUCIBILITY OF HEEGAARD SPLITTINGS OF SEIFERT FIBERED SPACES

ERIC SEDGWICK

Volume 190 No. 1

September 1999 


\title{
THE IRREDUCIBILITY OF HEEGAARD SPLITTINGS OF SEIFERT FIBERED SPACES
}

\author{
ERIC SEDGWICK
}

\begin{abstract}
Moriah and Schultens have demonstrated that an irreducible Heegaard splitting of an orientable Seifert fibered space over an orientable base surface is either vertical or horizontal. In this paper it is determined precisely which vertical and horizontal splittings are irreducible. Let $M$ be a Seifert fibered space which admits a horizontal splitting at the fiber $f$. If the genus of the horizontal splitting at $f$ is less than the genus of the vertical splittings, its genus will be minimal and the splitting irreducible. Otherwise, this splitting will be irreducible if and only if the multiplicity of the fiber $f$ is strictly greater than the least common multiple of the multiplicities of the other fibers. In particular, each Seifert fibered space possesses at most one irreducible horizontal splitting. The vertical splittings will be reducible if and only if $M$ has a horizontal splitting with genus strictly less than the genus of the vertical splittings.
\end{abstract}

\section{Introduction.}

Throughout this paper a Seifert fibered space, $M$, will denote a closed, orientable Seifert fibered space over an orientable base surface $B$. Then $M$ may be written $M=\left\{g_{0}, e_{0} \mid\left(\alpha_{1}, \beta_{1}\right),\left(\alpha_{2}, \beta_{2}\right), \ldots,\left(\alpha_{n}, \beta_{n}\right)\right\}$, where $g_{0}$ is the genus of the base surface $B, e_{0}$ is the Euler number of $M, n$ is the number of exceptional fibers, and $\left(\alpha_{i}, \beta_{i}\right)$ are the Seifert invariants of the exceptional fiber $f_{i}$. In particular, $\alpha_{i}$ denotes the multiplicity of the exceptional fiber $f_{i}$.

If $M$ has less than three exceptional fibers and base surface a 2-sphere then $M$ is $S^{3}$ or a lens space (or $S^{2} \times S^{1}$ ) and the irreducible Heegaard splittings of $M$ are classified by Waldhausen $[\mathbf{W}]$ and Bonahon and Otal [BO], respectively. ( $S^{3}$ has a unique horizontal splitting and lens spaces have a unique vertical splitting.) We therefore assume that $n \geq 3$ or $g_{0} \geq 1$.

The main results of $[\mathbf{M S}]$ and [Schu2] imply that irreducible Heegaard splittings of Seifert fibered spaces are vertical or horizontal. Every Seifert fibered space $M$ possesses vertical splittings and when $n \geq 2$ they may be thought of as a Heegaard splittings of $M$ which are also Heegaard splittings of the product manifold $M-N(E)$, where $N(E)$ is a neighborhood of the 
exceptional fibers. When $n<2$ it is necessary to also remove the neighborhood of one or two regular fibers. Nevertheless, each vertical splitting of $M$ will have the same genus, denoted $g_{v}$, and will be identified up to isotopy by a non-trivial partition of the exceptional fibers (and possibly one or two regular fibers).

A horizontal splitting exists if and only if the manifold $M$ can be described as a Dehn filling on a surface bundle $F \tilde{\times} S^{1}$, where the surface $F$ has a single boundary component, the bundle has finite monodromy and the meridian of the attached solid torus intersects the curve $\partial F$ exactly once. In this case the core of the attached solid torus will be a regular or exceptional fiber $f$ and we say that $M$ has a horizontal splitting at $f$. Necessary and sufficient conditions for this to occur are given in terms of the Seifert invariants in [MS]. The genus of the horizontal splitting at $f$ will be given by $g_{h}(f)=$ $2 g(F)$, where $g(F)$ is the genus of the surface $F$.

In this paper we will decide the irreducibility of the vertical and horizontal splittings. Typically, $g_{h}(f)$ will be greater than the genus of the vertical splittings $g_{v}$. When it is not the horizontal splitting will be called small. As the genus of a horizontal splitting is determined by a bundle structure on the complement of the fiber $f, M_{0}=M-N(f)$, the presence of a small horizontal splitting places restrictions on the manifold $M_{0}$, and hence upon the manifold $M$. These restrictions will be quantified by Theorem 5.3.

Theorem. Let $M$ admit a horizontal splitting at $f$. This splitting is small if and only if one the following holds:

$$
\begin{gathered}
M_{0}=\left\{g_{0} \mid \emptyset\right\}, g_{0} \geq 1, f \text { is regular or exceptional; } \\
M_{0}=\{0 \mid(2,1), \ldots,(2,1)\}, n_{0} \geq 3 \text { and odd, } f \text { is exceptional; } \\
M_{0}=\{0 \mid(2,1), \ldots,(2,1)\}, n_{0} \geq 3 \text { and odd, } f \text { is regular; } \\
M_{0}=\left\{0 \mid(2,1),\left(3, \beta_{2}\right)\right\}, f \text { is exceptional; } \\
M_{0}=\left\{0 \mid(2,1),\left(4, \beta_{2}\right)\right\}, f \text { is exceptional; } \\
M_{0}=\left\{0 \mid\left(3, \beta_{1}\right),\left(3, \beta_{2}\right)\right\}, f \text { is exceptional. }
\end{gathered}
$$

Furthermore, if (1) or (2) holds then $g_{h}(f)=g_{v}-1$. If one of (3)-(6) holds then $g_{h}(f)=g_{v}$.

This characterization will yield Corollary 5.5.

Corollary. A small horizontal splitting is of minimal genus and hence irreducible.

Non-small horizontal splittings are by definition not minimal genus and it is thus considerably more difficult to determine their irreducibility. This requires a close analysis of once punctured surface bundles and an application of a slight generalization of Casson's rectangle condition ([CG1]). The result of this analysis is Theorems 6.1 and 8.1, summarized as follows: 
Theorem. Let $M$ admit a non-small horizontal splitting at the fiber $f$. Then this splitting will be irreducible if and only if the multiplicity of the fiber $f$ is strictly greater than the least common multiple of the multiplicities of the other exceptional fibers.

From this theorem and the characterization in Theorem 5.3 we obtain Corollary 8.2.

Corollary. The Seifert fibered space $M$ admits an irreducible horizontal splitting at at most one fiber $f$.

Using the work of $[\mathbf{L M}],[\mathbf{M}]$, and $[\mathbf{S c h u 3}]$ the irreducibility of the vertical splittings is determined in Theorem 5.6.

Theorem. The vertical splittings will be reducible if and only if $M$ has a horizontal splitting with genus strictly less than the genus of the vertical splittings. In this case there is a horizontal splitting of genus one less than the vertical splittings.

The author gratefully acknowledges the helpful comments provided by Cameron Gordon and Jennifer Schultens.

\section{Preliminaries.}

The following notation and definitions are standard. For more information regarding Heegaard splittings see [RS], [ST2], or [Scha].

A handlebody is a homeomorph of a closed regular neighborhood of a connected graph in $S^{3}$. A Heegaard splitting of a closed orientable 3-manifold $M$ is a decomposition $M=H_{1} \cup_{G} H_{2}$, where $H_{1}$ and $H_{2}$ are handlebodies such that $\partial H_{1}=\partial H_{2}=H_{1} \cap H_{2}=G$. The genus of the Heegaard splitting is the genus of the surface $G$ and the genus of $M, g(M)$ is the least genus of all Heegaard splittings of $M$.

An essential disk in a manifold with boundary $M$ is a properly embedded disk $D$ such that $\partial D$ is an essential loop in $\partial M$. A Heegaard splitting is: stabilized if there are essential disks $D_{1} \subset H_{1}$ and $D_{2} \subset H_{2}$ such that $\partial D_{1} \cap \partial D_{2}=\{$ point $\}$, reducible if there are essential disks $D_{1} \subset H_{1}$ and $D_{2} \subset H_{2}$ such that $\partial D_{1}=\partial D_{2}$, and weakly reducible if there are essential disks $D_{1} \subset H_{1}$ and $D_{2} \subset H_{2}$ such that $\partial D_{1} \cap \partial D_{2}=\emptyset$.

\section{Vertical Heegaard Splittings.}

In this section we give a brief description of vertical Heegaard splittings of Seifert fibered spaces and state a few well known facts that will be needed in later sections. A more detailed treatment of vertical splittings is given in [BZ] (see also [MS] and [Schu1]).

By identifying each fiber to a point we obtain a projection, $\rho: M \rightarrow B$, from the Seifert fibered space onto the base surface $B$. If $f$ is a fiber then 
$e=\rho(f)$ is a point on the base surface $B$. We will call $e$ the anchor point corresponding to the fiber $f$.

A vertical Heegaard splitting of a Seifert fibered space is identified by choosing a non-trivial partition of the exceptional fibers contained in $M$. (If the number of exceptional fibers is less than two then we cannot form a non-trivial partition and must add 1 or 2 regular fibers to the exceptional fibers and then partition.) Choose a minimal graph $\Sigma$ in the base surface $B$ so that $B-\Sigma$ is a disk and $\Sigma$ is disjoint from the anchor points of the fibers in the partition. Take $\operatorname{arcs}$ on $B$ and adjoin to $\Sigma$ the anchor points of each of the fibers from the first half of the partition, and for all but one of the fibers in the second half, adjoin to $\Sigma$ a loop on $B$ encircling its anchor point. We may lift this graph to $M$ and attach to it the fibers from the first half of the partition. A closed regular neighborhood of the resulting graph, $\Sigma^{\prime}$, is clearly a handlebody. Moreover, the complement of an open regular neighborhood of $\Sigma^{\prime}$ is also a handlebody (see [Schu1]).

Definition 3.1. Let $\Sigma^{\prime}$ be obtained as above. The Heegaard splitting $M=$ $\overline{N\left(\Sigma^{\prime}\right)} \cup N\left(\Sigma^{\prime}\right)^{C}$ is called a vertical splitting of $M$.

In this manner we can construct vertical splittings for any Seifert fibered space. The isotopy class of the vertical splitting obtained will be well-defined up to the choice of partition, a fact established in $[\mathbf{L M}]$. However, the choice of a different partition produces a vertical splitting which may or may not be isotopic to the first. (A great deal is known about the equivalence of vertical splittings, see $[\mathbf{L}],[\mathbf{L M}]$, and $[\mathbf{M}]$.) As the genus of a vertical splitting is independent of the partition chosen and depends solely on the genus of the base surface, $g_{0}$, and the number of of exceptional fibers, $n$, all vertical splittings of $M$ have the same genus and we may define:

Definition 3.2. The vertical genus of $M, g_{v}$, is the genus of each of the vertical splittings.

The vertical genus was first calculated by Boileau and Zieschang ([BZ]) and is given by:

$$
\begin{aligned}
& \text { If } n<2 \text { then } g_{v}=2 g_{0}+1 . \\
& \text { If } n \geq 2 \text { then } g_{v}=2 g_{0}+n-1 .
\end{aligned}
$$

For "most" Seifert fibered spaces the vertical splittings will be of minimal genus and the vertical genus will coincide with the Heegaard genus of the manifold; the exceptions to this rule will be determined in Theorem 5.3 and Corollary 5.7. 


\section{Once Punctured Surface Bundles and Horizontal Heegaard Splittings.}

This section contains background material regarding the horizontal construction that will be essential in proving the main theorems.

Choose a fiber, $f$, (regular or exceptional) and remove a solid torus neighborhood of this fiber from $M$. Let $(\alpha, \beta)$ be the Seifert invariants of the fiber $f$. Then $M_{0}=M-N(f)=\left\{g_{0} \mid\left(\alpha_{1}, \beta_{1}\right), \ldots,\left(\alpha_{n_{0}}, \beta_{n_{0}}\right)\right\}$ is an orientable Seifert fibered space over an orientable base surface $B_{0}=B-N(p t)$ with $n_{0}$ exceptional fibers (where $n_{0}=n$ or $n-1$ ) and a single boundary component.

It is known ([J, VI.32]) that a Seifert fibered space with a single boundary component fibers as a periodic surface bundle over the circle, $M_{0}=F \tilde{\times} S^{1}$, where the fiber $F$ is a connected and orientable surface and the orbit of any point under the $S^{1}$ action is a fiber in the Seifert fibering. We can write $M_{0}=F \times I /(x \times\{1\} \sim h(x) \times\{0\})$ where $h: F \rightarrow F$ is the periodic homeomorphism associated with the bundle $M_{0}=F \tilde{\times} S^{1}$. The periodic homeomorphism $h$ will have degree $d=\operatorname{lcm}\left\{\alpha_{1}, \ldots, \alpha_{n_{0}}\right\}$. Define the equivalence, $x \sim y$, if $y=h^{i}(x)$ for some $i$. Let $[x]$ denote the equivalence class of $x$. Then the map $\rho: M_{0} \rightarrow B_{0}$ given by $\rho(x \times i)=[x]$ is the standard projection map of the Seifert fibered space $M_{0}$. If we restrict to $F, \rho: F \rightarrow B_{0}$ is a branched covering. Let $e_{1}, \ldots, e_{n_{0}}$ be the anchor points on $B_{0}$ of the exceptional fibers $f_{1}, \ldots, f_{n_{0}}$ which have multiplicities $\alpha_{1}, \ldots, \alpha_{n_{0}}$. Then $\rho: F \rightarrow B_{0}$ will have degree $d=\operatorname{lcm}\left\{\alpha_{1}, \ldots, \alpha_{n_{0}}\right\}$ and branch points $e_{1}, \ldots, e_{n_{0}}$ with corresponding branching indices $\alpha_{1}, \ldots, \alpha_{n_{0}}$. In Figure 1 there are two anchor points with multiplicities $\alpha_{1}=3, \alpha_{2}=2(d=6)$.

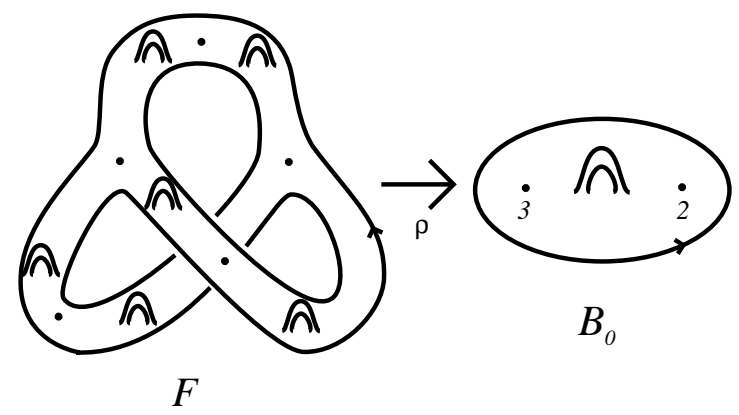

Figure 1. $\rho: F \rightarrow B_{0}$.

We state our first restriction: Consider only the case where the fiber $F$ has a single boundary component. Choose two disjoint copies of the surface fiber, $F_{1}$ and $F_{2}$, and cut along these surfaces to decompose $M_{0}=F \tilde{\times} S^{1}$ 
into two pieces, $F \times I_{1}$ and $F \times I_{2}$. Label the surfaces $F_{1}$ and $F_{2}$ and orient $I_{1}$ and $I_{2}$ so that $F \times I_{1}{ }^{-}=F_{1}, F \times I_{1}{ }^{+}=F_{2}, F \times I_{2}{ }^{-}=F_{2}$, and $F \times I_{2}{ }^{+}=F_{1}$. We may assume that the monodromy $h$ is the identity on $F \times I_{1}$ and is $h$ on $F \times I_{2}$. Note that each of these pieces is a handlebody, being a punctured surface cross an interval. The boundary of $M_{0}$ is decomposed into two annuli, $\partial M_{0}=A_{1} \cup A_{2}$ where $A_{i}$ is the annulus $\partial F \times I_{i}$.

We obtain $M$ by gluing the solid torus neighborhood of $f, \overline{N(f)}$, to the boundary of $M_{0}$. We now introduce the second restriction: The meridian $m$ of the solid torus $\overline{N(f)}$ must intersect $\partial F$ exactly once. Then $m_{1}=m \cap A_{1}$ and $m_{2}=m \cap A_{2}$ will each be a single arc and the manifold $M$ may be thought of as the quotient $M_{0} /\left(A_{1}=A_{2}\right)$ where the gluing of $A_{1}$ and $A_{2}$ is defined by identifying the $\operatorname{arcs} m_{1}$ and $m_{2}$. (See Figure 2.)

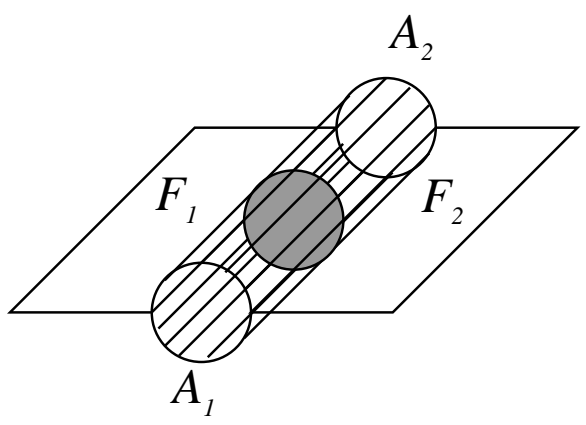

Figure 2. $M=M_{0} /\left(A_{1}=A_{2}\right)$.

We have expressed $M$ as the union of two handlebodies glued along their common boundary, this is a Heegaard splitting of $M$. If the surface fiber $F$ does not have a single boundary component or the meridian disk does not meet the boundary of the surface fiber exactly one time, the manifold $M$ will fail to have a horizontal Heegaard splitting corresponding to the fiber $f$. (However, it may have a horizontal splitting corresponding to removing the neighborhood of a different regular or exceptional fiber.) A test in terms of the Seifert invariants is given in [MS]. Assuming that the two restrictions are met we define:

Definition 4.1. The Heegaard splitting $M=\left(F \times I_{1}\right) \cup_{G}\left(F \times I_{2}\right)$, where $G=F_{1} \cup A_{1} \cup F_{2}=F_{2} \cup A_{2} \cup F_{1}$ is called a horizontal splitting of $M$ at $f$.

Note that the horizontal splitting at $f$ will be determined only up to homeomorphism as the fibration $M_{0}=F \tilde{\times} S^{1}$ is determined only up to homeomorphism of $M_{0}$. This author suspects (but has not proven) that some Seifert fibered spaces will possess an infinite number of non-isotopic but 
homeomorphic splittings obtained by twisting a given horizontal splitting in vertical tori.

We now calculate the genus of the horizontal splittings. This calculation was made in [MS] and is included here for the sake of completeness. Remove disk neighborhoods of the anchor points $e_{i}$ on $B_{0}$ to obtain $B_{0}^{\prime}=B_{0}-$ $N\left(\cup_{i=1, \ldots, n_{0}} e_{i}\right)$. Similarly remove the lifts of these neighborhoods from $F$ to obtain $F^{\prime}=F-N\left(\rho^{-1}\left(\cup_{i=1, \ldots, n_{0}} e_{i}\right)\right.$. The restriction, $\rho: F^{\prime} \rightarrow B_{0}^{\prime}$, is a (non-branched) covering of degree $d$, under which the Euler characteristic is multiplicative. Thus,

$$
\begin{aligned}
& \chi\left(B_{0}^{\prime}\right)=\chi\left(B_{0}\right)-n_{0}=1-2 g_{0}-n_{0} \\
& \chi\left(F^{\prime}\right)=d \chi\left(B_{0}\right)=d\left(1-2 g_{0}-n_{0}\right) .
\end{aligned}
$$

We now replace the disks that we removed from $F$ to get $F^{\prime}$. In doing so we observe that the disk containing $e_{i}$ will be branched covered by $d / \alpha_{i}$ disjoint disks each with branching index $\alpha_{i}$.

$$
\begin{aligned}
\chi(F) & =\chi\left(F^{\prime}\right)+\sum_{i=1}^{n_{0}} d / \alpha_{i} \\
& =d\left(1-2 g_{0}-n_{0}+\sum_{i=1}^{n_{0}} 1 / \alpha_{i}\right) .
\end{aligned}
$$

Let $g_{h}(f)$ denote the genus of a horizontal splitting at $f$. If $M$ admits a horizontal splitting at $f, g_{h}(f)$ will be twice the genus of the punctured surface $F$.

$$
\begin{aligned}
g_{h}(f) & =2 g(F)=1-\chi(F) \\
& =1+d\left(n_{0}+2 g_{0}-1-\sum_{i=1}^{n_{0}} 1 / \alpha_{i}\right) .
\end{aligned}
$$

If $M$ does not admit a horizontal splitting at $f$ define

$$
g_{h}(f)=\infty \text {. }
$$

Definition 4.2. Call $g_{h}=\min \left\{g_{h}(f) \mid \mathrm{f}\right.$ is a regular or exceptional fiber $\}$, the horizontal genus of the manifold $M$.

If $M$ does not admit a horizontal splitting $g_{h}=\infty$.

We will need a more concise description of $\partial M_{0}$ before the identification of $A_{1}$ and $A_{2}$ is made. We will denote by $\langle a, b\rangle$ the oriented intersection number of the curves $a$ and $b$ on $\partial M_{0}$.

Recall that the horizontal construction requires that the meridian $m$ of the solid torus $\overline{N(f)}$ meets $\partial F$ precisely once. That means that $(\partial F, m)$ is a basis for the homology of the boundary torus $\partial M_{0}$. We will use this coordinate system for the remaining discussion. Let $x \in \partial B_{0}$. Then $f_{r}=\rho^{-1}(x)$ is a 
regular fiber on $\partial M_{0}$. As $\rho$ restricts to a branched covering of degree $d$ on $F$ we may orient $\partial F$ such that $\left\langle\partial F, f_{r}\right\rangle=d$.

Furthermore, since $f$ is a fiber of multiplicity $\alpha$ we can orient $m$ so that $\left\langle f_{r}, m\right\rangle=\alpha$. Recall that we have restricted the monodromy $h$ to the piece $F \times I_{2}$, thus $f_{r}=\alpha \cdot \partial F+d \cdot m$ will appear as pictured in Figure 3 .

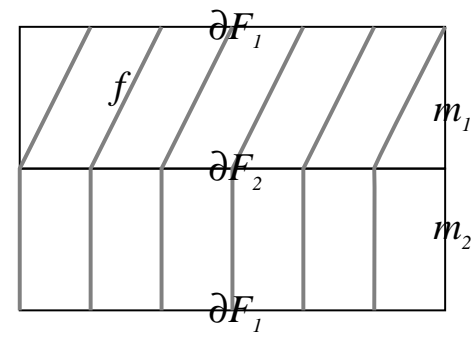

$\partial M_{0}$

Figure 3. $\partial M_{0}$ in $(\partial F, m)$ coordinates.

As the fiber $f_{r}$ consists of a single component, we have established the following lemma.

Lemma 4.3. Let $M$ admit a horizontal splitting at the fiber $f$. Then $\alpha$ and $d$ are relatively prime.

Additionally, since $d=\operatorname{lcm}\left\{\alpha_{1}, \ldots, \alpha_{n_{0}}\right\}$ we have:

Lemma 4.4. Let $M$ admit a horizontal splitting at the fiber $f$. Then $\alpha$ is relatively prime to the multiplicity of each of the other fibers.

Definition 4.5. A regular arc is an arc of the form $x \times I_{i}$ where $x \in \partial F_{i}$.

Define a metric on $\partial B_{0}$ so that the length of $\partial B_{0}$ is one and lift it to a metric on $\partial F$. With this metric the length of $\partial F$ is $d$.

A regular arc is a sub-arc of a regular fiber which lies in $\partial M_{0}$. In the $(\partial F, m)$ coordinate system a regular arc on $A_{1}$ will be vertical, whereas a regular arc on $A_{2}$ will have an $\alpha$ shift. In the following sections it will be relevant to compare the values $\alpha$ and $d$. If $\alpha>d$ then every regular arc on $A_{2}$ will necessarily intersect the $\operatorname{arc} m_{2}$. Conversely, if $\alpha<d$ then there is a regular arc on $A_{2}$ that will not intersect $m_{2}$.

\section{Small Horizontal Splittings and the Irreducibility of Vertical Splittings.}

In this section we classify the horizontal splittings which have genus less than the vertical splittings and demonstrate that they are irreducible. Additionally, using methods and results from [Schu1], [Schu3], and [LM] we determine under what conditions the vertical splittings are reducible. 
Definition 5.1. Let $M$ admit a horizontal splitting at the fiber $f$. If $g_{h}(f) \leq g_{v}$ then we say that this splitting is small.

A horizontal splitting is derived from a branched covering of the base surface and unless the degree of the covering, $d$, and the genus of the base surface, $g_{0}$, are small, we expect the genus of the horizontal splitting to be quite large, and in particular to exceed the genus of the vertical splittings. This statement is made precise in the following lemma.

Lemma 5.2. Let $M$ admit a horizontal splitting at $f$. This splitting is small if and only if one of the following statements holds:

1) $d=1$.

2) $d=2$ and $g_{0}=0$.

3) $g(F)=1$ and $\left(g_{0}=0\right)$.

Proof. There are three cases according to the value of $d$.

Case. $d=1$.

When $d=1, n_{0}=0$ and $n \leq 1$. The horizontal genus is

$$
g_{h}(f)=1+1\left(0+2 g_{0}-1\right)=2 g_{0}
$$

and the vertical genus is given by

$$
g_{v}=2 g_{0}+1 \text {. }
$$

Thus,

$$
g_{h}(f)=g_{v}-1
$$

and the result follows in this case.

Case. $d=2$.

When $d=2$ we have that for all $i=1, \ldots, n_{0}, \alpha_{i}=2$ and therefore $\sum_{i=1}^{n_{0}} 1 / \alpha_{i}=n_{0} / 2$. The horizontal genus is then

$$
g_{h}(f)=1+2\left(n_{0}+2 g_{0}-1-n_{0} / 2\right)=n_{0}+4 g_{0}-1 .
$$

If $n \geq 2$ then $g_{v}=2 g_{0}+n-1 \leq 2 g_{0}+n_{0}$. There is at least one multiplicity two fiber in $M_{0}$, hence $n_{0} \geq 1$. If $n<2$ then $g_{v}=2 g_{0}+1 \leq 2 g_{0}+n_{0}$. Therefore,

$$
g_{v} \leq 2 g_{0}+n_{0}
$$

Then $g_{h}(f) \leq g_{v}$ if and only if

$$
\begin{aligned}
n_{0}+4 g_{0}-1 & \leq 2 g_{0}+n_{0} \\
g_{0} & \leq 1 / 2 \\
g_{0} & =0,
\end{aligned}
$$

as $g_{0}$ is a non-negative integer. This establishes the result for the case $d=2$. 
Case. $d \geq 3$.

When $d \geq 3$ we have that $\alpha_{i} \geq 2$ for every $i$ and therefore $\sum_{i=1}^{n_{0}} 1 / \alpha_{i} \leq n_{0} / 2$. The horizontal genus is then

$$
\begin{aligned}
g_{h}(f) & \geq 1+d\left(n_{0}+2 g_{0}-1-\sum_{i=1}^{n_{0}} 1 / \alpha_{i}\right) \\
& \geq 1+3\left(n_{0} / 2+2 g_{0}-1\right)=3 / 2 n_{0}+6 g_{0}-2 .
\end{aligned}
$$

As in the previous case,

$$
g_{v} \leq 2 g_{0}+n_{0}
$$

Then $g_{h}(f) \leq g_{v}$ if and only if

$$
\begin{aligned}
3 / 2 n_{0}+6 g_{0}-2 & \leq 2 g_{0}+n_{0} \\
n_{0}+4 g_{0} & \leq 2 .
\end{aligned}
$$

It follows that $g_{0}=0$ and $n_{0} \leq 3$. Recalling that $g_{h}(f)=2 g(F)$ we further calculate

$$
\begin{aligned}
2 g(F)=g_{h}(f) & \leq g_{v} \leq 2 g_{0}+n_{0} \\
2 g(F) & \leq 3 \\
g(F) & \leq 3 / 2 .
\end{aligned}
$$

As $M$ is not $S^{3}$ or a lens space we always have that $g_{h}(f) \geq 2$ and therefore $g(F) \geq 1$. We conclude that $g(F)=1$. By the same reasoning we always have $g_{v} \geq 2$. Thus, when $g(F)=1$ we have that $g_{h}(f) \leq g_{v}$. This establishes the result for the case $d \geq 3$.

Combining the previous lemmas with some basic facts about horizontal splittings we can give a list of the small horizontal splittings.

Theorem 5.3. Let $M$ have a horizontal splitting at $f$. This splitting is small if and only if one the following holds:

$$
\begin{gathered}
M_{0}=\left\{g_{0} \mid \emptyset\right\}, g_{0} \geq 1, f \text { is regular or exceptional; } \\
M_{0}=\{0 \mid(2,1), \ldots,(2,1)\}, n_{0} \geq 3 \text { and odd, } f \text { is exceptional; } \\
M_{0}=\{0 \mid(2,1), \ldots,(2,1)\}, n_{0} \geq 3 \text { and odd, } f \text { is regular; } \\
M_{0}=\left\{0 \mid(2,1),\left(3, \beta_{2}\right)\right\}, f \text { is exceptional; } \\
M_{0}=\left\{0 \mid(2,1),\left(4, \beta_{2}\right)\right\}, f \text { is exceptional; } \\
M_{0}=\left\{0 \mid\left(3, \beta_{1}\right),\left(3, \beta_{2}\right)\right\}, f \text { is exceptional. }
\end{gathered}
$$

Furthermore, if (1) or (2) holds then $g_{h}(f)=g_{v}-1$. If one of (3)-(6) holds then $g_{h}(f)=g_{v}$.

Proof. There are three cases according to the value of $d$ : 
Case. $d=1$.

Then $n_{0}=0$ and $n=0$ or 1 . As $M$ is not $S^{3}$ or a lens space, it follows that $g_{0} \geq 1$. This is (1) where we can readily calculate that

$$
\begin{aligned}
g_{v} & =2 g_{0}+1 \\
g_{h}(f) & =1+d\left(n_{0}+2 g_{0}-1-\sum_{i=1}^{n_{0}} 1 / \alpha_{i}\right)=2 g_{0} \\
g_{h}(f) & =g_{v}-1 .
\end{aligned}
$$

Case. $d=2$.

It follows from Lemma 5.2 that $g_{0}=0$. Then $M_{0}=\{0 \mid(2,1),(2,1), \ldots$, $(2,1)\}$ and we note that

$$
\begin{aligned}
\chi(F) & =d\left(\sum_{i=1}^{n_{0}} 1 / \alpha_{i}-n_{0}+1-2 g_{0}\right) \\
& =2\left(n_{0} / 2-n_{0}+1-0\right)=2-n_{0} .
\end{aligned}
$$

If $n_{0}$ is even the surface $F$ will have more than one boundary component and the horizontal construction will fail. Thus $n_{0}$ is odd. Since $g_{0}=0$ we require $n \geq 3$. Then

$$
\begin{aligned}
g_{v} & =2 g_{o}+n-1=n-1 \\
g_{h}(f) & =1+d\left(n_{0}+2 g_{0}-1-\sum_{i=1}^{n_{0}} 1 / \alpha_{i}\right) \\
& =1+2\left(n_{0}-1-n_{0} / 2\right)=n_{0}-1 .
\end{aligned}
$$

Either $f$ is an exceptional fiber, $n=n_{0}+1$ and

$$
g_{h}(f)=n=g_{v}-1,
$$

or $f$ is regular, $n=n_{0}$ and

$$
g_{h}(f)=n-1=g_{v} .
$$

These are the cases (2) and (3).

Case. $d \geq 3$.

It follows from Lemma 5.2 that $g(F)=1$ and $g_{0}=0$. As $M$ is not a lens space or $S^{3}, n \geq 3$ and $n_{0} \geq 2$. Also each $\alpha_{i} \geq 2$ implies $\sum_{i=1}^{n_{0}} 1 / \alpha_{i} \leq n_{0} / 2$. We have 


$$
\begin{aligned}
& g_{h}(f)=2 g(F) \\
& 1+d\left(n_{0}+2 g_{0}-1-\sum_{i=1}^{n_{0}} 1 / \alpha_{i}\right)=2 \\
& 1+3\left(n_{0} / 2-1\right) \leq 2 \\
& n_{0} \leq 8 / 3 \text {. }
\end{aligned}
$$

Hence $n_{0}=2, f$ is an exceptional fiber and $n=3$.

It is now demonstrated that $d \leq 6$. Suppose that $d>6$. Then one of $\alpha_{1}, \alpha_{2}$ is strictly larger than 3 , say $\alpha_{1}>3$. Then

$$
\begin{gathered}
2=1+d\left(2-1-1 / \alpha_{1}-1 / \alpha_{2}\right)>1+6\left(1-1 / 3-1 / \alpha_{2}\right) \\
1 / 6>1-1 / 3-1 / \alpha_{2} \\
2>\alpha_{2} \\
\alpha_{2}=1 .
\end{gathered}
$$

This is a contradiction, and we conclude that $d \leq 6$.

As $d=\operatorname{lcm}\left(\alpha_{1}, \alpha_{2}\right)$ it follows that $\left(\alpha_{1}, \alpha_{2}\right)$ is one of $(2,2),(2,3),(2,4)$, $(2,6),(3,3),(3,6),(4,4),(5,5),(6,6)$. Calculate $\chi(F)$ for:

$$
\begin{aligned}
& (2,2): \chi(F)=d\left(\sum_{i=1}^{n_{0}} 1 / \alpha_{i}-n_{0}+1-2 g_{0}\right)=2(1 / 2+1 / 2-2+1)=0 ; \\
& (2,6): \chi(F)=6(1 / 2+1 / 6-2+1)=-2 ; \\
& (3,6): \chi(F)=6(1 / 3+1 / 6-2+1)=-3=1-2 g(F) \Rightarrow g(F)=2 ; \\
& (4,4): \chi(F)=4(1 / 4+1 / 4-2+1)=-2 \\
& (5,5): \chi(F)=5(1 / 5+1 / 5-2+1)=-3=1-2 g(F) \Rightarrow g(F)=2 ; \\
& (6,6): \chi(F)=6(1 / 6+1 / 6-2+1)=-4
\end{aligned}
$$

For the pairs $(2,2),(2,6),(4,4)$ and $(6,6)$ the Euler characteristic $\chi(F)$ is even, so $F$ has more than one boundary component and the horizontal construction fails. For $(3,6)$ and $(5,5) g(F)=2$ which contradicts our assumptions. We are left with the cases where the pair $\left(\alpha_{1}, \alpha_{2}\right)=(2,3),(2,4)$ or $(3,3)$. The horizontal construction can indeed work in these cases. This is (4), (5) or (6) with the $\alpha$ 's listed.

In these cases:

$$
\begin{aligned}
g_{v} & =2 g_{0}+n-1=2 \\
g_{h}(f) & =2 g(F)=2 \\
g_{h}(f) & =g_{v}=2 .
\end{aligned}
$$


We can now refer to a small horizontal splitting as being one of type (1)-(6). Using this characterization, we can easily prove

Theorem 5.4. $M$ admits a small horizontal splitting at at most one fiber $f$.

Proof. Seeking a contradiction, suppose that $M$ admits distinct small horizontal splittings at $f$ and $f^{\prime}$. We know from Lemma 4.4 that the corresponding multiplicities $\alpha$ and $\alpha^{\prime}$ are relatively prime.

Suppose that one of the splittings, say the one at $f$, is a type (1) small splitting, i.e.

$$
M_{0}=\left\{g_{0} \mid \emptyset\right\}, g_{0} \geq 1, f \text { is regular or exceptional. }
$$

As $f^{\prime}$ belongs to $M_{0}$ it must be a regular fiber. Furthermore, $f$ must be an exceptional fiber or these splittings are both at regular fibers and are not distinct. Corresponding to the splitting at $f^{\prime}$ we have

$$
M_{0}^{\prime}=\left\{g_{0} \mid(\alpha, \beta)\right\}, g_{0} \geq 1, f^{\prime} \text { is regular. }
$$

But then $d^{\prime}=\alpha>1$, as $f$ is not regular, and $g_{0} \geq 1$ together imply that the splitting at $f^{\prime}$ is not small. This is a contradiction.

Next, suppose that no pair of the fibers in $M_{0}$ are relatively prime. Then, no matter how we select $\alpha^{\prime}$ it will never be relatively prime to $d^{\prime}$. This prevents $M$ from possessing two distinct horizontal splittings (small or not) where one is a small splitting of type $(2),(3),(5)$ or (6).

The remaining case is when both the splitting at $f$ and the splitting at $f^{\prime}$ are type (4),

$$
M_{0}=M_{0}^{\prime}=\left\{0 \mid(2,1),\left(3, \beta_{2}\right)\right\}, f \text { is exceptional. }
$$

Again, this is impossible, as $\alpha$ would have to be 2 or 3 so that $M_{0}^{\prime}$ appeared as a type (4) splitting. This contradicts the fact that $\alpha$ and $d$ are relatively prime.

Finally this establishes:

Corollary 5.5. A small horizontal splitting is of minimal genus and hence irreducible.

Proof. Minimal genus splittings are always irreducible. If $M$ admits a small horizontal splitting at $f$, then it must be of minimal genus. If there were a splitting of smaller genus, which we may assume is irreducible, by [MS] it would either be horizontal or vertical. It cannot be vertical, given the hypothesis that the splitting at $f$ is small. Nor can it be horizontal, for this would imply that $M$ admitted two distinct small horizontal splittings in direct contradiction with the previous lemma. 
The following theorem determines the irreducibility of the vertical splittings. Although this particular phrasing may be new, the theorem is essentially a corollary of the earlier work of Lustig, Moriah and Schultens ([LM], [Schu1] and [Schu3]).

Theorem 5.6. The following statements hold:

(i) $g_{h}<g_{v}$ if and only if $M$ admits a small horizontal splitting of type (1) or (2) if and only if the vertical splittings are reducible.

(ii) $g_{h}=g_{v}$ if and only if $M$ admits a small horizontal splitting of type (3)-(6). If so, then the vertical splittings are irreducible.

(iii) $g_{h}>g_{v}$ implies that the vertical splittings are irreducible.

Proof. We start with statement (i). Theorem 5.3 establishes that $g_{h}<g_{v}$ if and only if $M$ admits a type (1) or (2) small horizontal splitting. If the vertical splittings are reducible then there must be a splitting of smaller genus and by $[\mathbf{M S}]$ it is horizontal and we have that $g_{h}<g_{v}$.

The more difficult implication is that when $g_{h}<g_{v}$ the vertical splittings are reducible. The argument is due to Schultens ([Schu3]). Let $M$ admit a small horizontal splitting of type (1) or (2). Then $M$ has a unique (up to isotopy) vertical splitting. If this splitting is type (1) then $n \leq 1$ and there will be only one non-trivial partition of a collection of 2 fibers. If the splitting is type (2) then $\beta=1$ for all but at most one fiber in $M$ (the fiber not of multiplicity 2). As demonstrated in the proof of [LM] Theorem $2.8 M$ then admits only one vertical splitting. (If $f$ is a fiber with $\beta=1$ then there is an isotopy between any two vertical splittings whose partitions differ only by $f$.) In [Schu3] Schultens shows that a stabilization of a horizontal splitting at $f$ is a splitting of the manifold $M_{0}$. In [Schu1] it is also shown that irreducible splittings of manifolds with boundary are vertical. It follows that in this case the stabilization of a horizontal splitting at $f$ coincides with the unique vertical splitting of $M$. Thus the vertical splitting is a stabilization of the unique horizontal splitting (in $M$, not in $\left.M_{0}\right)$ and is reducible.

Statement (ii) is cases (3)-(6) of Theorem 5.3. As the vertical splittings are of minimal genus they are irreducible.

In the case of statement (iii) the vertical splittings are of minimal genus and hence irreducible.

The following corollary addresses the Heegaard genus of Seifert fibered spaces. Most of this problem was solved in [BZ]; the few remaining cases follow from the results in [MS]. We include it here for completeness.

Corollary 5.7. The Heegaard genus of a Seifert fibered space $M$ is equal to the vertical genus unless $M$ admits a type (1) or (2) small horizontal 
splitting. In the latter case, the Heegaard genus of $M$ is one less than the vertical genus.

\section{Reducibility of Horizontal Splittings.}

In this section we will prove the first half of the main theorem.

Theorem 6.1. Let $M$ admit a horizontal splitting at $f$ which is not small. If $\alpha<d$ then this splitting is reducible.

The proof is postponed until later in this section. Casson and Gordon demonstrated that a weakly reducible Heegaard splitting is either reducible or the manifold in question contains an incompressible surface. Although Seifert fibered spaces usually contain incompressible surfaces, in [Schu2] Schultens demonstrates that weakly reducible horizontal splittings are indeed reducible. We therefore seek to prove that these horizontal splittings are weakly reducible. The following lemma will be our primary tool in establishing the theorem. Recall that an essential arc in a surface $F$ is a properly embedded arc which does not cobound a disk on $F$ with an arc on $\partial F$.

Lemma 6.2. Let $M$ admit a horizontal splitting at a fiber $f$ so that $\alpha<d$. If there are essential arcs $\gamma_{1}$ and $\gamma_{2}$ in $F$ such that

(i) $\gamma_{1} \cap\left(\gamma_{2} \cup h\left(\gamma_{2}\right)\right)=\emptyset$, and

(ii) There is an interval $I \subset \partial F$ of length less than 1 that contains, in sequence, both of the endpoints of $\gamma_{1}$ followed by both of the endpoints of $\gamma_{2}$. (The orientation on I is given by the orientation on $\partial F$.), then this splitting is weakly reducible (and hence reducible).

Proof. Note that we do not require that $\gamma_{2} \cap h\left(\gamma_{2}\right)=\emptyset$.

Claim. The length of $I$ can be taken to be arbitrarily small.

The endpoints of $h\left(\gamma_{2}\right)$ are a positive integer distance from the endpoints of $\gamma_{2}$ and thus cannot be contained in the interval $I$ of length less than one. Therefore we may choose a neighborhood of $I$ which is disjoint from $h\left(\gamma_{2}\right)$. Within this neighborhood we can isotope the arcs so that their endpoints are arbitrarily close together. This can be done while maintaining $\gamma_{1} \cap\left(\gamma_{2} \cup\right.$ $\left.h\left(\gamma_{2}\right)\right)=\emptyset$.

Define the product disks $D_{1}=\gamma_{1} \times I_{1}$ and $D_{2}=\gamma_{2} \times I_{2}$. These disks are essential in the handlebodies $H_{1}=F \times I_{1}$ and $H_{2}=F \times I_{2}$. We now check that the boundaries of these disks are disjoint on the splitting surface $G=F_{1} \cup A_{1} \cup F_{2}=F_{2} \cup A_{2} \cup F_{1}$ by analyzing the intersection $\partial D_{1} \cap \partial D_{2}$ as restricted to each of the pieces, $F_{1}, F_{2}$ and $A=A_{1}=A_{2}$.

On $F_{1}$ we have $D_{1} \cap F_{1}=\gamma_{1}$ and $D_{2} \cap F_{1}=h\left(\gamma_{2}\right)$ which are disjoint by hypothesis. Similarly on $F_{2}, D_{1} \cap F_{2}=\gamma_{1}$ and $D_{2} \cap F_{2}=\gamma_{2}$ are disjoint by assumption. 
Before we make the identification $A=A_{1}=A_{2}$ we note that the boundary of $D_{1}$ on $\partial M_{0}$ is two regular arcs on $A_{1}$ which are vertical and that the boundary of $D_{2}$ on $\partial M_{0}$ is two regular arcs on $A_{2}$ which have an $\alpha$ shift. See Figure 4. We may take the endpoints of the arcs to be arbitrarily close together. In particular as $\alpha<d$ we may make them close enough so that after identifying $A_{1}$ to $A_{2}$ the regular arcs on $A_{1}$ will not intersect the regular arcs on $A_{2}$. Therefore, $\partial D_{1} \cap \partial D_{2}=\emptyset$ and the splitting is weakly reducible.

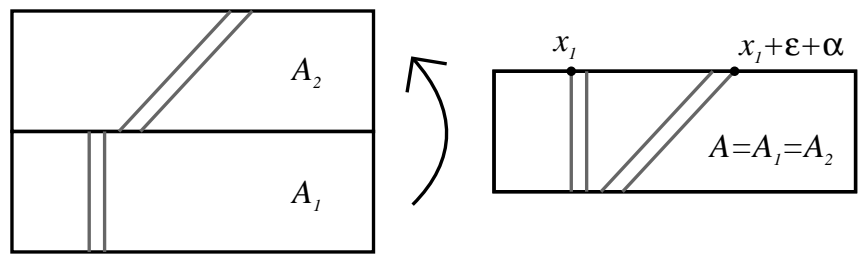

Figure 4. The regular arcs will not intersect after identifying $A_{1}$ and $A_{2}$.

With Lemma 6.2, all that is needed to prove the main theorem of the section is to show that for a non-small horizontal splitting we can always find such arcs $\gamma_{1}$ and $\gamma_{2}$. However, we know that we will never be able to produce such arcs for a small splitting (or else we could make it reducible). Unfortunately, this forces the proof of the main theorem to be quite technical; it must make exact use of the properties of non-small horizontal splittings.

Proof of Theorem 6.1. The horizontal splitting being considered is not small, according to our characterization in Lemma 5.2 this leaves us with two possibilities, either

1) $g_{0} \geq 1$, or

2) $d \geq 3$ and $g(F)>1$.

We will prove these two cases separately.

Case. $g_{0} \geq 1$.

In this case we can find a non-separating essential curve on the base surface $B_{0}$. Furthermore, we may take two disjoint copies of this curve, $\phi_{1}$ and $\phi_{2}$, and adjoin to them embedded arcs, $\tau_{1}$ and $\tau_{2}$ (resp.), which attach them to $\partial B_{0}$ and so that regular neighborhoods of each of the resulting graphs are disjoint from each other and from the anchor points of the exceptional fibers. See Figure 5. As the total length of $\partial B_{0}$ is equal to 1 , the distance between the endpoint of $\tau_{1}$ and $\tau_{2}$ is strictly less than 1 .

Recall that $\rho: F \rightarrow B$ is a branched covering map. We may consider the preimages of our chosen graphs and neighborhoods on $F$. The preimage, $\rho^{-1}\left(\tau_{i}\right)$, of the arc $\tau_{i}$ will be $d$ disjoint copies of $\tau_{i}$. For each $i$ choose 

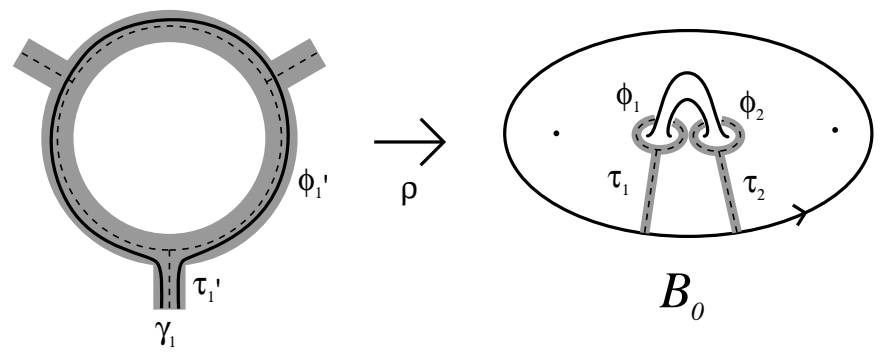

Figure 5. Lift neighborhoods of the graphs to $F$.

an arc $\tau_{i}^{\prime} \subset \rho^{-1}\left(\tau_{i}\right)$ so that the distance from the endpoint of $\tau_{1}$ to the endpoint of $\tau_{2}^{\prime}$ is a positive number less than 1. Each of these arcs joins a closed loop $\phi_{i}{ }^{\prime} \subset \rho^{-1}\left(\phi_{i}\right)$ to $\partial F$. For $i=1,2$ choose regular neighborhoods $N\left(\phi_{i}{ }^{\prime} \cup \tau_{i}{ }^{\prime}\right) \subset \rho^{-1}\left(N\left(\phi_{i} \cup \tau_{i}\right)\right)$. One component of $\partial\left(N\left(\tau_{i}{ }^{\prime} \cup \phi_{i}{ }^{\prime}\right)\right)$ will be an $\operatorname{arc} \gamma_{i}$ (the other component is a curve). As $\phi_{i}{ }^{\prime}$ is essential and non-boundary parallel, $\gamma_{i}$ is an essential arc on $F$. By taking the regular neighborhood to be sufficiently small we have that the endpoints of $\gamma_{1}$ are followed by those of $\gamma_{2}$ and all are contained in an interval of length less than one. The arcs then satisfy hypothesis ii) of Lemma 6.2.

Moreover, $\gamma_{1}$ is disjoint from both $\gamma_{2}$ and $h\left(\gamma_{2}\right)$. To see this note that $\gamma_{1} \subset \rho^{-1}\left(N\left(\phi_{1} \cup \tau_{1}\right)\right)$ and $\gamma_{2}, h\left(\gamma_{2}\right) \subset \rho^{-1}\left(N\left(\phi_{2} \cup \tau_{2}\right)\right)$. In turn, these sets are disjoint being preimages of disjoint neighborhoods on $B_{0}$. Therefore, the essential arcs $\gamma_{1}$ and $\gamma_{2}$ satisfy the conditions of Lemma 6.2. In this case the horizontal splitting at $f$ is weakly reducible.

We now to proceed to the second (and more difficult) case.

Case. $d \geq 3$ and $g(F)>1$.

We assume that $g_{0}=0$ (otherwise the result follows from the previous case). Now, there are no essential loops or arcs on the base surface that we can lift to $F$ and we must construct $\gamma_{1}$ and $\gamma_{2}$ by more laborious means.

Definition 6.3. An almost essential arc on the base surface $B_{0}$ is a properly embedded arc on $B_{0}$ which is also an essential arc on the punctured surface $B_{0}{ }^{\prime}=B_{0}-N\left(\cup_{i=1, \ldots, n_{0}} e_{i}\right)$, where the $e_{i}$ are the anchor points of exceptional fibers contained in $M_{0}$.

Note that the preimage under $\rho$ of an almost essential arc on $B_{0}$ will be a collection of essential arcs on $F$. 
Choose an almost essential arc $\gamma$ on the base surface $B_{0}$ and choose an arc $\gamma_{1} \subset \rho^{-1}(\gamma)$ on $F$. Apply the surface homeomorphism $h$ iteratively to obtain the essential arcs $\gamma_{2}=h\left(\gamma_{1}\right)$, and $\gamma_{3}=h\left(\gamma_{2}\right)$.

Claim. $\gamma_{i}$ and $\gamma_{i+1}$ are disjoint and not parallel.

Suppose that $\gamma_{i}$ and $\gamma_{i+1}$ are parallel. Then every arc in $\Gamma=\rho^{-1}(\gamma)$ will be parallel and $\Gamma$ will contain at least $3 \operatorname{arcs}(d \geq 3)$. From $\Gamma$ choose three parallel and adjacent arcs: $\gamma_{a}, \gamma_{b}$ and $\gamma_{c}$. Let $R_{1}$ be the disk region bounded by $\gamma_{a}$ and $\gamma_{b}$ and $R_{2}$ be the disk region bounded by $\gamma_{b}$ and $\gamma_{c}$. We know that $\gamma$ separates the disk $B_{0}$ into two subdisks $B_{1}$ and $B_{2}$ and $\rho$ restricted to $R_{1}$ is a branched covering of one of $B_{1}$ or $B_{2}$, say $B_{1}$. It follows that $\rho$ restricted to $R_{2}$ is a branched covering of $B_{2}$. This implies that each of the disk regions $R_{i}$ is a double branched cover of the corresponding $B_{i}$. So $B_{1}$ and $B_{2}$ each contain exactly one branch point of multiplicity 2 and $d=2$, a contradiction.

Consider $F-\left(\gamma_{1} \cup \gamma_{2} \cup \gamma_{3}\right)$. We have assumed that $g(F)>1$ and the previous claim shows that neither $\gamma_{1}$ nor $\gamma_{3}$ is parallel to $\gamma_{2}$. Thus, $\gamma_{2}$ is on the boundary of a non-disk region $E$ of $F-\left(\gamma_{1} \cup \gamma_{2} \cup \gamma_{3}\right)$. We may choose an essential arc $\delta \subset E \subset F$ with endpoints at $x_{2}+2 \epsilon$ and $x_{2}+3 \epsilon$ $(0<\epsilon<1 / 3)$ where $x_{2}$ is one of the endpoints of $\gamma_{2}$. (See Figure 6.) Then $h(\delta)$ is disjoint from $\gamma_{2}, \gamma_{3}$ and $\gamma_{4}$. So $\delta$ and $h(\delta)$ may not be disjoint, but, $(\delta \cup h(\delta)) \cap\left(\gamma_{2} \cup \gamma_{3}\right)=\emptyset$. Let $x_{3}=h\left(x_{2}\right)$, the endpoints of $h(\delta)$ will be $x_{3}+2 \epsilon$ and $x_{3}+3 \epsilon$.

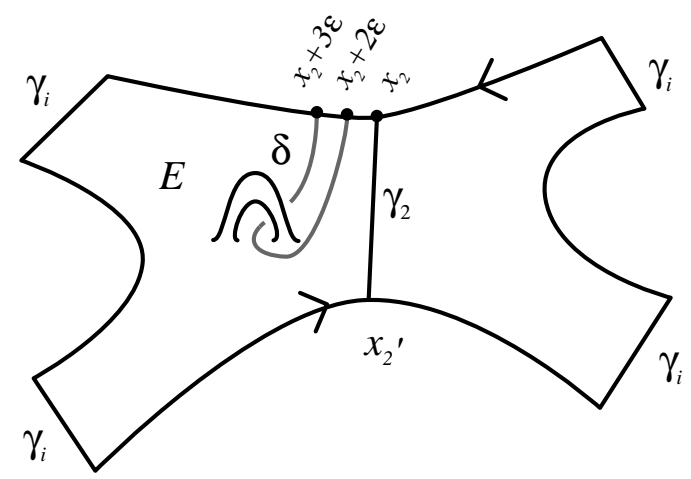

Figure 6. Choosing the $\operatorname{arc} \delta$.

Claim. Let $R$ be the component of $F-(\delta \cup h(\delta))$ that contains the point $x_{2}$. Then $R$ is not a disk.

Suppose that $R$ is a disk. From Figure 7 we can see that $\partial F-(\delta \cup h(\delta))$ consists of four interval components. Let $J_{2}$ be the interval containing $x_{2}$, $J_{2}=\left(x_{3}+3 \epsilon, x_{2}+2 \epsilon\right)$, and $J_{3}$ be the interval containing $x_{3}, J_{3}=\left(x_{2}+\right.$ 
$\left.3 \epsilon, x_{3}+2 \epsilon\right)$. Note that $R$ contains the entire arc $\gamma_{2}$ and $J_{2} \subset \partial R$ (and possibly $J_{3} \subset \partial R$ ).

Suppose both endpoints of $\gamma_{2}, x_{2}$ and $x_{2}{ }^{\prime}$, lie on the interval $J_{2}$. That would imply that the arc $\gamma_{2}$ is inessential which is a contradiction.

Therefore $x_{2}^{\prime}$ lies on the interval $J_{3}$ and the disk $R$ contains the arc $\gamma_{3}$ as well. Then $\gamma_{3}$ must have also have an endpoint on each of $J_{2}$ and $J_{3}$ (or it is inessential). But then, $\gamma_{2}$ and $\gamma_{3}$ lie in the disk $R$ and have adjacent endpoints on the intervals $J_{2}$ and $J_{3}$ and are thus parallel on the surface $F$. This is a contradiction, hence $R$ is not a disk.
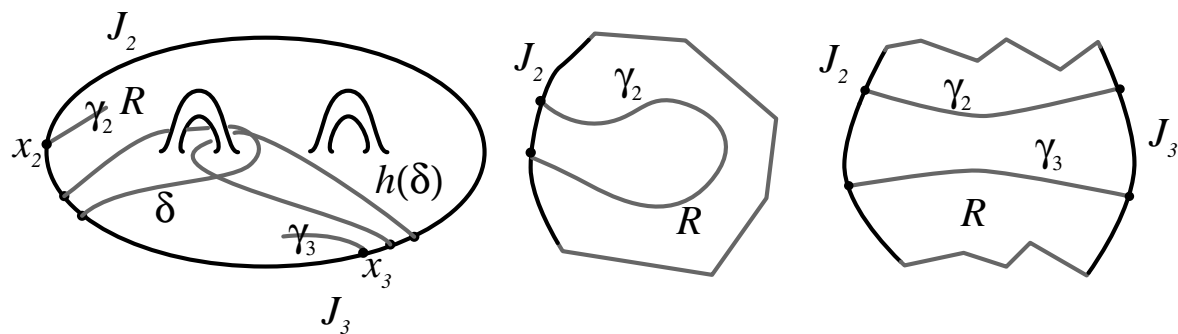

Figure 7. $R$ is not a disk.

As $R$ is not a disk we can find an essential $\operatorname{arc} \zeta \subset R$ with endpoints at $x_{2}$ and $x_{2}+\epsilon$. So $\zeta$ is an essential arc on $F$ that is disjoint from $\delta \cup h(\delta)$.

The endpoints of $\delta$ are at $x_{2}+2 \epsilon$ and $x_{2}+3 \epsilon$ and $\epsilon<1 / 3$. Then the arcs $\zeta$ and $\delta$ satisfy the hypotheses of Lemma 6.2 and a horizontal splitting at $f$ is weakly reducible.

\section{The Rectangle Condition.}

Non-small horizontal splittings are by definition not of minimal genus. To prove their irreducibility it must be directly shown that any pair of essential disks, one in each handlebody, intersect at least twice. In [CG1], Casson developed the rectangle condition which allows one to analyze the intersection of any pair of disks by working with prechosen disk systems. Casson's rectangle condition was given by a pair of pants decomposition of a surface, here it is generalized to a planar decomposition.

Definition 7.1. Let $G$ be a closed surface and $\mathcal{C} \subset G$ a collection of pairwise disjoint curves. Then $\mathcal{C}$ is a complete curve system for $G$ if $\mathcal{P}=G-\mathcal{C}$ is a collection of planar surfaces, none of which is a disk or an annulus. We call $\mathcal{P}$ the induced planar decomposition. 
Definition 7.2. Let $\Delta$ be a collection of disjoint essential disks in a handlebody $H$, no two of which are parallel. If $H-\Delta$ is a collection of balls then we call $\Delta$ a complete collection of disks for $H$.

Note that if $\Delta$ is a complete collection of disks for a handlebody $H$ then $\partial \Delta$ is a complete collection of curves for $G=\partial H$ and hence induces a planar decomposition of $G$.

Definition 7.3. Let $P$ be a planar surface. Suppose that $c \subset \partial P$ is a single curve and $C_{1} \subset \partial P$ and $C_{2} \subset \partial P$ are non-empty and disjoint collections of curves such that $\partial P=c \sqcup \mathcal{C}_{1} \sqcup \mathcal{C}_{2}$. We will call the decomposition $\partial P=c \sqcup \mathcal{C}_{1} \sqcup \mathcal{C}_{2}$ a non-trivial partition of $\partial P$.

Definition 7.4. Let $P$ and $P^{\prime}$ be planar surfaces embedded on a closed surface $G$. If for each non-trivial partition of $\partial P=c \sqcup \mathcal{C}_{1} \sqcup \mathcal{C}_{2}$ and each non-trivial partition of $\partial P^{\prime}=c^{\prime} \sqcup \mathcal{C}_{1}{ }^{\prime} \sqcup \mathcal{C}_{2}{ }^{\prime}$ there exist curves $c_{1} \in C_{1}, c_{2} \in$ $C_{2}, c_{1}{ }^{\prime} \in C_{1}{ }^{\prime}$, and $c_{2}{ }^{\prime} \in C_{2}{ }^{\prime}$ such that $P \cap P^{\prime}$ contains a rectangle bounded by $c_{1}, c_{2}, c_{1}{ }^{\prime}$, and $c_{2}{ }^{\prime}$ then $P$ and $P^{\prime}$ are said to satisfy the rectangle condition for planar surfaces.

Definition 7.5. Let $\mathcal{C}$ and $\mathcal{C}^{\prime}$ be collections of curves on a surface $G$. Then $\mathcal{C}$ and $\mathcal{C}^{\prime}$ intersect essentially if no component of $G-\left(\mathcal{C} \cup \mathcal{C}^{\prime}\right)$ is a bigon, a disk region bounded by two arcs.

Definition 7.6. Suppose that $\mathcal{C}$ and $\mathcal{C}^{\prime}$ are complete collections of curves that intersect essentially on a surface $G$ and that $\mathcal{P}$ and $\mathcal{P}^{\prime}$ are their respective induced planar decompositions. Then $\mathcal{C}$ and $\mathcal{C}^{\prime}$ are said to satisfy the rectangle condition for curve systems if for each $P \in \mathcal{P}$ and each $P^{\prime} \in \mathcal{P}^{\prime}, P$ and $P^{\prime}$ satisfy the rectangle condition for planar surfaces.

Definition 7.7. Let $M=H \cup_{G} H^{\prime}$ be a Heegaard splitting. Let $\Delta$ and $\Delta^{\prime}$ be complete collections of disks for $H$ and $H^{\prime}$, respectively. Further assume that $\partial \Delta \subset G$ and $\partial \Delta^{\prime} \subset G$ intersect essentially and that $\mathcal{P}$ and $\mathcal{P}^{\prime}$ are their respective induced planar decompositions. If for each choice of $P \in \mathcal{P}$ and $P^{\prime} \in \mathcal{P}^{\prime}, P$ and $P^{\prime}$ satisfy the rectangle condition for planar surfaces then we say that $\Delta$ and $\Delta^{\prime}$ satisfy the rectangle condition for disk systems.

Lemma 7.8. Let $H \cup_{F} H^{\prime}$ be a Heegaard splitting. Let $D \subset H$ and $D^{\prime} \subset$ $H^{\prime}$ be essential disks. If there are complete collections of disks, $\Delta \subset H$ and $\Delta^{\prime} \subset H^{\prime}$, which satisfy the rectangle condition for disk systems then $\left|\partial D \cap \partial D^{\prime}\right| \geq 4$. In particular, the splitting $H \cup_{F} H^{\prime}$ is strongly irreducible.

Proof. First isotope the disks $D$ and $D^{\prime}$ so that the quantity $\mid \partial D \cap \partial D^{\prime} \cap$ $\partial \Delta \cap \partial \Delta^{\prime} \mid$ is minimal.

Claim. No two curves in the collection $\partial D \cup \partial D^{\prime} \cup \partial \Delta \cup \partial \Delta^{\prime}$ intersect inessentially. 
If so, then there is an innermost bigon (other arcs may be contained in the bigon, but they join distinct boundary arcs of the bigon). As $\partial \Delta \cap \partial \Delta^{\prime}$ is essential by the definition of the rectangle condition, one of these disks must be $D$ or $D^{\prime}$, say $\mathrm{D}$. The bigon can be used to construct an isotopy of $D$ that reduces $\left|\partial D \cap \partial D^{\prime} \cap \partial \Delta \cap \partial \Delta^{\prime}\right|$. But, we have assumed that this quantity is minimal. The claim holds.

As there are no innermost bigons, there are no bigons at all and the curves $\partial D$ and $\partial D^{\prime}$ must intersect minimally. Furthermore, as handlebodies are irreducible we may isotope the interiors of the disk so that there are no closed loops of intersection in $D \cap \Delta$ and $D^{\prime} \cap \Delta^{\prime}$.

Consider an outermost subdisk $D_{0}$ of $D-\Delta$. Let $\delta$ designate the portion of $\partial D$ that is coincident with $\partial D_{0}$. Either $D_{0} \subsetneq D$ and $\delta$ is an arc or $D_{0}=D$ and $\delta=\partial D$, a loop. See Figure $8 . \delta$ is properly embedded in a single component $P \in \mathcal{P}$.

Claim. $\delta$ is an essential loop or arc.

If $\delta$ is an arc and inessential then it forms a bigon with some $c \in \partial P$ and there is an inessential intersection which contradicts the previous claim. If $\delta$ is a loop and inessential then the disk $D$ is inessential, again a contradiction.

Similarly, we can find an essential arc (or loop) $\delta^{\prime}$ that lies entirely and essentially in a planar piece $P^{\prime}$.

Case. $\delta$ and $\delta^{\prime}$ are both arcs.

As $\delta$ belongs to an outermost disk both of its endpoints belong to a single loop $c \in \partial P$. As $\delta$ is essential in $\partial P$ it separates the remaining curves, $\partial P-c$, into two non-empty sets $C_{1}$ and $C_{2}$. We obtain the non-trivial partition $\partial P=c \sqcup C_{1} \sqcup C_{2}$. Similarly $\delta^{\prime}$ yields the non-trivial partition $\partial P^{\prime}=c^{\prime} \sqcup C_{1}{ }^{\prime} \sqcup C_{2}{ }^{\prime}$ where $\delta^{\prime}$ separates $C_{1}{ }^{\prime}$ from $C_{2}{ }^{\prime}$.

By assumption $\mathcal{C}$ and $\mathcal{C}^{\prime}$ satisfy the rectangle condition for curve systems which in turn implies that the pieces $P$ and $P^{\prime}$ satisfy the rectangle condition for planar surfaces. Thus there exist curves $c_{1} \in C_{1}, c_{2} \in C_{2}, c_{1}{ }^{\prime} \in C_{1}{ }^{\prime}$, and $c_{2}{ }^{\prime} \in C_{2}{ }^{\prime}$ which bound a rectangle on $P \cap P^{\prime}$. As $\delta$ separates $c_{1}$ and $c_{2}$ on $P$ (it separates $C_{1}$ from $C_{2}$ ) and $\delta^{\prime}$ separates $c_{1}{ }^{\prime}$ and $c_{2}{ }^{\prime}$ on $P^{\prime}$, the $\operatorname{arcs} \delta$ and $\delta^{\prime}$ must meet in the rectangle. See Figure 8.

Any such outermost arc $\delta$ must meet any outermost arc $\delta^{\prime}$ at least once. There will be at least two outermost subdisks on $D$ and thus we obtain at least two distinct choices for $\delta$. Similarly there are at least two distinct choices for $\delta^{\prime}$. For each pair we obtain a distinct point of intersection. Thus $\partial D \cap \partial D^{\prime} \geq 4$.

Case. $\delta$ is a loop and $\delta^{\prime}$ is an arc.

As $\delta$ is essential and entirely contained in $P$ it separates $\partial P$ into two collections $C_{1}$ and $C_{2}$. One collection, say $C_{1}$, contains more than one curve ( $P$ is not an annulus). Select $c \in C_{1}$ and form the non-trivial partition 

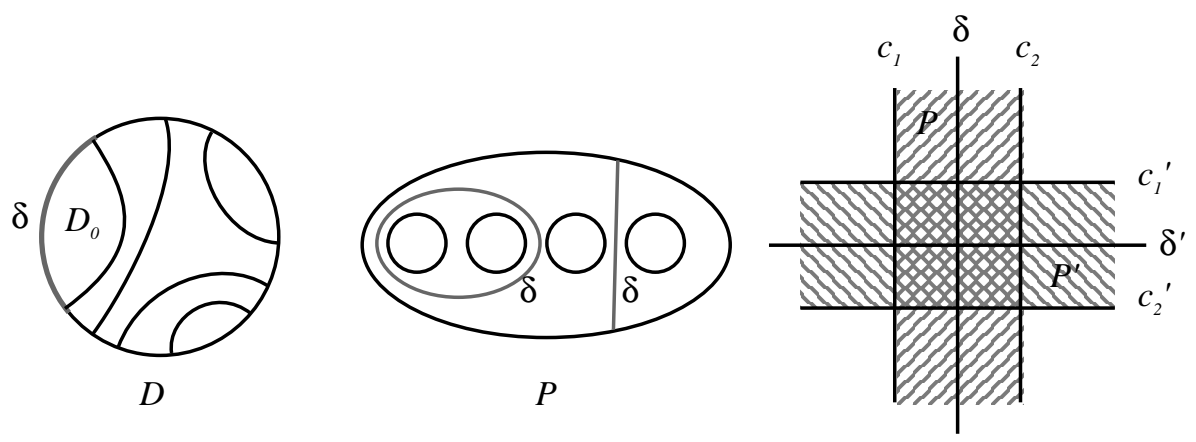

Figure 8. $\delta$ and $\delta^{\prime}$ must meet in the rectangle.

$\partial P=c \sqcup\left(C_{1}-c\right) \sqcup C_{2} . \delta$ separates the curves in $C_{1}-c$ from those in $C_{2}$. As before the arc $\delta^{\prime}$ will induce a non-trivial partition $\partial P^{\prime}=c^{\prime} \sqcup C_{1}{ }^{\prime} \sqcup C_{2}{ }^{\prime}$ where it separates separates $C_{1}{ }^{\prime}$ from $C_{2}{ }^{\prime}$.

As our curve systems satisfy the rectangle condition, we can find curves $c_{1} \in C_{1}, c_{2} \in C_{2}, c_{1}{ }^{\prime} \in C_{1}{ }^{\prime}$, and $c_{2}{ }^{\prime} \in C_{2}{ }^{\prime}$ which bound a rectangle on $P \cap P^{\prime}$. Again $\delta$ will separate $c_{1}$ and $c_{2}$ on $P$ and $\delta^{\prime}$ will separate $c_{1}{ }^{\prime}$ and $c_{2}{ }^{\prime}$ on $P^{\prime}$. Then $\delta$ and $\delta^{\prime}$ will have a point of intersection $x_{1}$.

Let $\delta_{1}{ }^{\prime}=\delta^{\prime} \cap P$ be the subarc of $\delta^{\prime}$ that contains the point $x_{1}$. Then $\delta_{1}{ }^{\prime}$ is a properly embedded arc on $P$ that joins the curves $c_{1}$ and $c_{2}$. See Figure 8.

Note that $\delta$ also separates the curves in $C_{1}-c_{1}$ (non-empty) from the curves in $C_{2}$ and we obtain the non-trivial partition $\partial P=c_{1} \sqcup\left(C_{1}-c_{1}\right) \sqcup C_{2}$. Then there are curves $d_{1} \in C_{1}-c_{1}, d_{2} \in C_{2}$ and $d_{1}{ }^{\prime} \in C_{1}{ }^{\prime}$, and $d_{2}{ }^{\prime} \in C_{2}{ }^{\prime}$ which bound a rectangle on $P \cap P^{\prime}$. Because $\delta$ and $\delta^{\prime}$ separate the curves in the chosen non-trivial partitions they must meet at a point $x_{2}$ in this rectangle.

Let $\delta_{2}{ }^{\prime}=\delta^{\prime} \cap P$ be the subarc of $\delta^{\prime}$ that contains the point $x_{2}$. Then $\delta_{2}{ }^{\prime}$ is a properly embedded arc on $P$ that joins the curves $d_{1}$ and $d_{2}$. Note that $c_{1} \neq d_{1}\left(d_{1} \in C_{1}-c_{1}\right)$ so the subarcs $\delta_{1}{ }^{\prime}$ and $\delta_{2}{ }^{\prime}$ are distinct. Therefore the points of intersection $x_{1}$ and $x_{2}$ are distinct.

As before there is a distinct choice for $\delta^{\prime}$ that will provide two additional points of intersection. Again, $\left|\partial D \cap \partial D^{\prime}\right| \geq 4$.

Case. $\delta$ and $\delta^{\prime}$ are both loops.

We proceed as in the previous case. For each loop there are at least two choices of a non-trivial partition. There are four combinations each yielding a point of intersection. Again, we can check that the points of intersection are distinct by checking that they are contained in different subarcs of $\partial \Delta \cap$ $P^{\prime}$ and $\partial \Delta^{\prime} \cap P$. 


\section{Irreducibility of Horizontal Splittings.}

In this section we complete the proof of the main theorem using the rectangle condition.

Theorem 8.1. Let $M$ admit a horizontal splitting at $f$. If $\alpha>d$ then this splitting is irreducible.

Before proving this theorem we will note and prove the following corollary.

Corollary 8.2. A Seifert fibered space admits an irreducible horizontal splitting at at most one fiber $f$.

Proof. Suppose that $M$ admits two irreducible splittings, at distinct fibers $f$ and $f^{\prime}$ with multiplicities $\alpha$ and $\alpha^{\prime}$. We have already demonstrated (Theorem 5.4) that both of these splittings cannot be small.

Nor can it be that neither splitting is small. In that case, our condition for irreducibility implies that $\alpha>\operatorname{lcm}\left\{\alpha_{i}\right\}_{i=1, \ldots, n_{0}}$. In particular $\alpha>\alpha^{\prime}$. Symmetrically $\alpha^{\prime}>\alpha$. This is a contradiction.

We then suppose that one of the splittings, say the one at $f$, is a small splitting and the other, at $f^{\prime}$, is not small. We may assume that $\alpha \leq \alpha^{\prime}$ (otherwise the above argument works).

If the splitting at $f$ is a type (1) splitting then

$$
M_{0}=\left\{g_{0} \mid \emptyset\right\}, g_{0} \geq 1, f \text { is regular or exceptional, }
$$

and in particular $\alpha^{\prime}=1$. Now $\alpha \leq \alpha^{\prime}$ implies that $\alpha=1$. Then both $f$ and $f^{\prime}$ are regular fibers and these two splittings are the same.

In the proof of Theorem 5.4 it was demonstrated that if $M$ admits a small splitting of type $(2),(3),(5)$, or (6) then this is the only horizontal splitting that $M$ admits. The remaining case is when there is a type (4) small horizontal splitting at $f$. So

$$
M_{0}=\left\{0 \mid(2,1),\left(3, \beta_{2}\right)\right\}, f \text { is exceptional. }
$$

We know that $\alpha \leq \alpha^{\prime}=2$ or 3. Moreover, $\alpha$ is relatively prime to $d=6$ and therefore $\alpha=1$. This contradicts the fact that $f$ is an exceptional fiber ( $M$ is not a lens space).

We now proceed with the proof of the second half of the main theorem.

Proof of Theorem 8.1. Let $E \subset B_{0}$ be the collection of anchor points of exceptional fibers. Let $E_{2}$ be the sub collection of multiplicity two anchor points. Choose the following collections of disjoint embedded arcs in $B_{0}$.

$\Gamma_{1}=\left\{2 g_{0}\right.$ arcs chosen such that $B_{0}-\Gamma_{1}$ is a single disk $\}$

$\Gamma_{2}=\left\{\left|E_{2}\right|\right.$ arcs, each joining a multiplicity two anchor point to a point on $\left.\partial B_{0}\right\}$. These arcs will be called half arcs. 
When $E_{2} \subsetneq E$ let $\Gamma_{3}=\left\{|E|-\left|E_{2}\right|-1\right.$ arcs so that $B_{0}-\Gamma_{1}-\Gamma_{2}-\Gamma_{3}$ is a collection of disks each of which contains exactly one point from the collection $\left.E-E_{2}\right\}$.

When $E_{2}=E$ let $\Gamma_{3}=\{\emptyset\}$.

These collections can be chosen so that the union $\Gamma_{0}=\Gamma_{1} \cup \Gamma_{2} \cup \Gamma_{3}$ is an embedded disjoint collection of arcs in $B_{0}$. With the exception of the arcs in $\Gamma_{2}$ these arcs are properly embedded in $B_{0}$.

Let $\Gamma=\rho^{-1}\left(\Gamma_{0}\right)$. Each arc in $\Gamma$ is essential on $F$. If the anchor points are all of multiplicity two then $B_{0}-\Gamma_{0}$ is a single disk which contains no branch points. Otherwise it is a collection of disks each containing exactly one branch point of multiplicity greater than or equal to 3 . It follows that $F-\Gamma_{0}$ is a collection of disks. Furthermore,

Claim. No two arcs in $\Gamma$ are parallel.

Let $\gamma_{a}$ and $\gamma_{b}$ be adjacent parallel arcs and $R$ be the component of $F-\Gamma$ bounded by them. Along $\partial R$ we see two arcs and two intervals of $\partial F$. The branched covering $\rho: F \rightarrow B_{0}$ restricts to a branched covering, $\rho: R \rightarrow R_{0}$, for some component $R_{0}$ in $B_{0}-\Gamma_{0}$. If $E \neq E_{2}$ then $R_{0}$ contains an anchor point of multiplicity at least three and $R$ has at least three arcs on its boundary, a contradiction. Therefore, all branch points are multiplicity two and $R_{0}$ is the sole component of $B_{0}-\Gamma_{0}$. Also, there are no branch points in the interior of $R_{0}$, so $R$ is a copy of $R_{0}$. But along $\partial R_{0}$ we see $2\left|\Gamma_{1}\right|+\left|\Gamma_{2}\right|=4 g_{0}+\left|E_{2}\right|$ arcs (the half arcs are doubled to look like full arcs). It follows that $g_{0}=0$ and $E=E_{2}=2$. So $F$ is a branched cover over the disk with two branch points each of multiplicity two. Then $F$ is an annulus, and does not have a single boundary component, contradicting the horizontal construction.

Let $\Delta_{1}=\Gamma \times I_{1}$ and $\Delta_{2}=\Gamma \times I_{2}$ be disk systems for $H_{1}=F \times I_{1}$ and $H_{2}=F \times I_{2}$ respectively. As no arc $\gamma \in \Gamma$ is inessential, each disk $D \in \Delta_{i}$ is essential. Moreover, as no two arcs in $\Gamma$ are parallel, no two disks in $\Delta_{i}$ are parallel. Each piece of $H_{i}-\Delta_{i}$ is a $\{$ disk $\} \times I$, in other words a ball. Thus $\Delta_{i}$ is a complete collection of disks for $H_{i}$.

Note that the boundaries of the disk collections are not transverse on the surfaces $F_{1}$ and $F_{2}$ but are coincident there. In fact $\partial \Delta_{1} \cap \partial \Delta_{2} \cap F_{1}$ (and similarly for $F_{2}$ ) is the collection of arcs $\Gamma$. By perturbing each disk in $\Delta_{2}$ slightly we may arrange that each coincident arc becomes a single point of intersection between $\Delta_{1}$ and $\Delta_{2}$ (See Figure 9). We treat this coincident arc as a single point of intersection and refer to it as a a long crossing.

By examining how the disk systems $\Delta_{1}$ and $\Delta_{2}$ intersect on the splitting surface $G=F_{1} \cup A_{1} \cup F_{2}=F_{2} \cup A_{2} \cup F_{1}$ it can be seen that they satisfy the rectangle condition.

Claim. The disk collections $\partial \Delta_{1}$ and $\partial \Delta_{2}$ intersect essentially. 


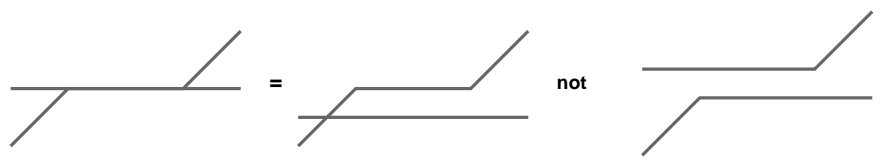

Figure 9. The disks intersect on $F_{i}$ in a long crossing.

If the disk collections $\partial \Delta_{1}$ and $\partial \Delta_{2}$ do not intersect essentially then some component of $G-\partial \Delta_{1} \cup \partial \Delta_{2}$ is a bigon. There are no components of $G-\partial \Delta_{1} \cup \partial \Delta_{2}$ lying entirely on the surfaces $F_{i}$ so we focus on $A=A_{1}=A_{2}$. See Figure 10. Each disk in $\Delta_{i}$ is of the form $\{\operatorname{arc}\} \times I_{i}$. Thus $\partial \Delta_{i} \cap$ $A_{i}$ is a collection of regular arcs. On $A$ the components of $G-\partial \Delta_{1} \cup$ $\partial \Delta_{2}$ are quadrilaterals contained on $A$ or triangular regions that extend onto one the surfaces $F_{i}$. The components of the first type are not bigons. The components of the second type already have three crossings, a regular crossing and two long crossings. Such a component cannot be a bigon unless its long crossings are coincident. This is impossible, the bigon would then imply that one of the arcs in $\Gamma$ (the long crossing) is inessential on $F_{i}$. Hence, there are no innermost bigons, and the intersection between $\partial \Delta_{1}$ and $\partial \Delta_{2}$ is minimal.
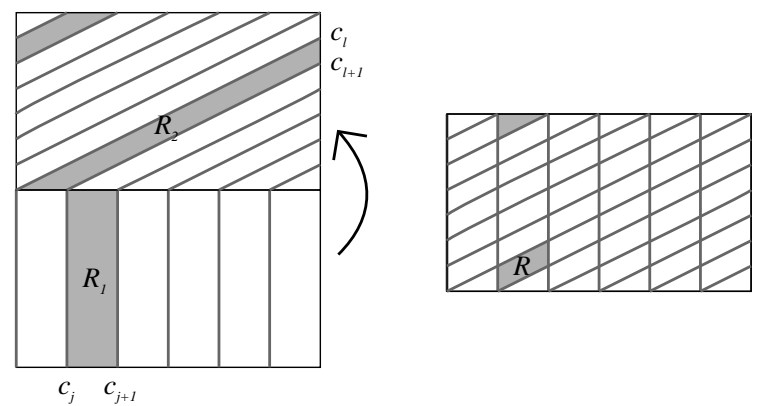

Figure 10. $\partial M_{0}$ when $\alpha>d$.

Let $P_{1}$ be any component of $\partial H_{1}-\Delta_{1}$ and $\partial P_{1}=c \sqcup C_{1} \sqcup C_{2}$ any non-trivial partition of its boundary components. Then $P_{1}=R \times I_{1}$ for some component $R$ of $F-\Gamma$ and appears as in Figure 11. By following $\partial R$ around we may cyclically label the arcs in $\partial R \cap \Gamma$ as they are encountered. Each component $c$ of $\partial P_{1}$ corresponds to one of these arcs and we can thus cyclically label the components of $\partial P_{1}, \partial P_{1}=\left\{c_{1}, c_{2}, \ldots, c_{m}\right\}$. As the components are cyclically ordered there must be consecutive boundary components $c_{j} \in C_{1}$ and $c_{j+1} \in C_{2}$ (or vice-versa). Select the rectangle $R_{1}$ which is the subset 
of the annulus $A_{1}$ that is bounded by these adjacent curves. In the same fashion given any planar piece $P_{2}$ and a non-trivial partition of its boundary curves we choose a rectangle $R_{2}$ bounded by adjacent curves, $c_{l}$ and $c_{l+1}$, that belong to different components of the partition.

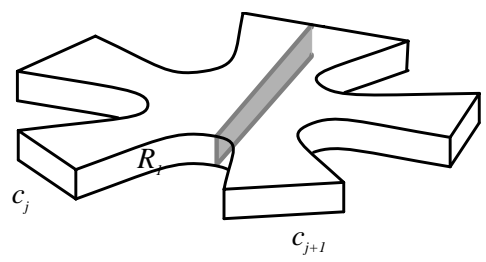

Figure 11. A piece of $F \times I_{i}-\Delta_{i}$.

Consider $\partial M_{0}=A_{1} \cup A_{2}$ as pictured in Figure 10. The rectangle $R_{1}$ is bounded by adjacent regular arcs on $A_{1}$ and is thus a vertical strip. The rectangle $R_{2}$ is also bounded by adjacent regular arcs and is therefore a strip with an $\alpha$ shift.As $\alpha>d$, after the identification $A_{1}=A_{2}$ is made, $R_{1} \cup R_{2}$ will contain a rectangle $R$.

The rectangle $R$ is contained in $R_{1} \cap R_{2} \subset P_{1} \cap P_{2}$ and is bounded by the curves $c_{j}, c_{j+1}, c_{l}$ and $c_{l+1}$ that were selected from different components of each of the given partitions. As the planar pieces and partitions were chosen arbitrarily, we have demonstrated that the disk collections $\Delta_{1}$ and $\Delta_{2}$ satisfy the rectangle condition, hence this horizontal splitting is irreducible.

\section{References}

[BZ] M. Boileau and Zieschang, Heegaard genus of closed orientable Seifert 3-manifolds, Inventiones Math., 76 (1984), 455-468.

[BO] F. Bonahon and J.P. Otal, Scindements de Heegaard des espaces lenticulaires, Ann. Sci. de l'ecole Norm. Sup, 16 (1983), 451-467.

[CG1] A. Casson and C. Gordon, Manifolds with irreducible splittings of arbitrarily high genus, unpublished.

[CG2] - Reducing Heegaard splittings, Topology and its Applications, 27 (1987), $275-283$.

[Ha] W. Haken, Some results on surfaces in 3-manifolds, M.A.A., Prentice Hall, (1968), 34-98.

[H] P. Heegaard, Forstudier til en topologisk teori for de algebraiske fladers sammenhaeng, Ph.D. thesis, Copenhagen, 1898.

[J] W. Jaco, Lectures on three-manifold topology, CBMS Regional Conference Series in Math, AMS, 43 (1980). 
[K] T. Kobayashi, A construction of 3-manifolds whose homeomorphism classes of Heegaard splittings have polynomial growth, Osaka Journal of Mathematics, 29 (1992), 653-674.

[L] M. Lustig, Nielsen equivalence and simple-homotopy type, Proc. London Math Society, 62(3) (1991), 537-562.

[LM] M. Lustig and Y. Moriah, Nielsen equivalence in Fuchsian groups and Seifert fibered spaces, Topology, 30 (1991), 451-467.

[M] Y. Moriah, Heegaard splittings of Seifert fibered spaces, Invent. Math., 91 (1988), 465-481.

[MS] Y. Moriah and J. Schultens, Irreducible Heegaard splittings of Seifert fibered spaces are horizontal or vertical, to appear in Topology.

[RS] H. Rubinstein and M. Scharlemann, Comparing Heegaard splittings of non-Haken 3-Manifolds, to appear in Topology.

[Scha] M. Scharlemann, Heegaard splittings of compact 3-manifolds, preprint.

[ST1] M. Scharlemann and A. Thompson, Heegaard splittings of (Surface) $\times I$ are standard, Math Ann., 295 (1993), 549-564.

[ST2] Thin position and Heegaard splittings of the 3-sphere, to appear in Jour. Diff. Geom.

[Schu1] J. Schultens, Heegaard splittings of Seifert fibered spaces with boundary, Transactions AMS, 347(7), July 1995.

[Schu2] — Weakly reducible Heegaard splittings of Seifert fibered spaces, (1994), preprint.

[Schu3] _ The stabilization problem for Heegaard splittings of Seifert fibered spaces, to appear in Topology and its Applications.

[W] F. Waldhausen, Heegaard - Zerlegungen der 3-sphare, Topology, 7 (1968), 195-203.

Received December 15, 1997 and revised May 10, 1998.

Oklahoma State University

STILLWATER, OK 74078

E-mail address: sedgwic@math.okstate.edu 